\title{
The Sommerfeld Half-Plane Problem Revisited, II The Factoring of a Matrix of Analytic Functions*)
}

\author{
A. E. Heins, Ann Arbor \\ Communicated by E. Meister
}

\begin{abstract}
A half-plane under plane wave excitation obeys a Dirichlet boundary condition on one side and a Neumann boundary condition on the other. These boundary conditions contrast the ones used by $\mathrm{A}$. Sommerfeld in his classical paper. The present problem leads to a system of integral equations of the Wiener-Hopf type which may be solved by a matrix factoring method suggested by A. E. Heins in 1950 .
\end{abstract}

\section{Introduction}

We return to our earlier paper [4] which dealt with a solution of the Rawlins' problem which we have described in part I. There we remarked that we did not have the necessary tools to solve this problem directly by the method of Wiener and Hopf. Indeed we had to devise a special method which was a combination of the methods of Wiener and Hopf and Carleman to provide the required solution. In the present paper, we now show that we can apply an idea which we put forth in 1948 [2] for the factoring of matrices of analytic functions and produce the required factors of the matrix which arises in the Rawlins' case. That this can be done is due to its special character and in fact it opens the path to solving a much more complicated problem in parallel plate media which we shall report in a later paper.

Basically, some of the problems which are now encountered in diffraction theory give rise to $2 \times 2$ matrices which have special algebraic properties and such problems were not noted in the late 1940's. Daniele [1] has repeated the original argument of Heins and discussed briefly two problems treated by Hurd and Przeździecki [5], [6]. We shall see that once the algebraic problem is solved we are basically left with a scalar problem in the present case.

Since we have already formulated this problem in part I, we shall refer to Sections II and III of that paper for the details and concentrate on the method for factoring the matrix. Though rudimentary in detail in the present case, our discussion will now point the way to treat the problem of a two dimensional

\footnotetext{
*) These results were announced at the XXth General Assembly of the U.R.S.I. held in Washington, D.C., August 1981.
} 
plane wave of arbitrary angle of incidence upon an infinite set of equally spaced, parallel plates which satisfies the Rawlins' type of boundary conditions.

II The Matrix Form of Equations (3.1) and (3.2) in Part I

We found in part $I$ that

$$
\frac{\mathrm{i}}{\sqrt{k^{2}-w^{2}}} \hat{f}_{1}(w)-\hat{f}_{2}(w)+\frac{2}{\mathrm{i}\left(w-k_{x}\right)}=2 \hat{F}_{2}(w)
$$

and

$$
-f_{1}(w)+\mathrm{i} \sqrt{k^{2}-w^{2}} f_{2}(w)+\frac{2 k_{y}}{\left(w-k_{x}\right)}=2 \hat{F}_{1}(w)
$$

Note that we have changed the enumeration of $\hat{F}_{1}(w)$ and $\hat{F}_{2}(w)$ from that used in part I. Here $f_{1}(w)$ is analytic in the lower half-plane $\operatorname{Im} w<\operatorname{Im} k_{x}$ while $f_{2}(w)$ is analytic when $\operatorname{Im} w<\operatorname{Im} k$. We also have that $\hat{F}_{1}(w)$ and $\hat{F}_{2}(w)$ are analytic in the upper half-plane $\operatorname{Im} w>-\operatorname{Im} k$, while $1 /\left(w-k_{x}\right)$ is analytic in the lower half-plane $\operatorname{Im} w<\operatorname{Im} k_{x}$. (Recall that $k$ has a positive imaginary part.) Equations (2.1) and (2.2) may be written in matrix form as

$$
A(w) f(w)-\frac{2}{\left(w-k_{x}\right)}\left(\begin{array}{c}
k_{y} \\
-\mathrm{i}
\end{array}\right)=-2 \hat{F}(w)
$$

where $A(w)=\left(\begin{array}{cc}1 & -\mathrm{i} \sqrt{k^{2}-w^{2}} \\ \frac{-\mathrm{i}}{\sqrt{k^{2}-w^{2}}} & 1\end{array}\right)$,

$$
\hat{f}(w)=\left(\begin{array}{l}
\hat{f}_{1}(w) \\
\hat{f}_{2}(w)
\end{array}\right) \text { and } \hat{F}(w)=\left(\begin{array}{l}
\hat{F}_{1}(w) \\
\hat{F}_{2}(w)
\end{array}\right) .
$$

The determinant of $A(w)$ is 2 and its elements are analytic in the strip - $\operatorname{Im} k$ $<\operatorname{Im} w<\operatorname{Im} k$.

We will now show that $A(w)$ may be factored such that $A_{+}(w) A_{-}(w)$ $=A_{-}(w) A_{+}(w)=A(w)$ where the elements of $A_{+}(w)$ are analytic in the upper half-plane $\operatorname{Im} w>-\operatorname{Im} k$, while those of $A_{-}(w)$ are analytic in the lower half-plane $\operatorname{Im} w<\operatorname{Im} k$. In both cases the corresponding determinants are constant. In general, the determinants of $A_{+}(w)$ and $A_{-}(w)$ are not constant. We start by noting that the characteristic values of $A(w)$ are $\lambda_{1}=1-\mathrm{i}$ and $\lambda_{2}=1+\mathrm{i}$, a situation which doesn't arise very often. Hence we can write

$$
A(w)=\exp [B(w)]
$$


by Sylvester's theorem where

$$
\begin{aligned}
B(w) & =\frac{1}{2}\left[\left(\begin{array}{cc}
1 & -\sigma \\
-\frac{1}{\sigma} & 1
\end{array}\right]\left(\ln \sqrt{2}+\frac{\mathrm{i} \pi}{4}\right)+\left(\begin{array}{cc}
1 & \sigma \\
\frac{1}{\sigma} & 1
\end{array}\right]\left(\ln \sqrt{2}-\frac{\mathrm{i} \pi}{4}\right)\right] \\
& =I \ln \sqrt{2}-\frac{\mathrm{i} \pi}{4}\left(\begin{array}{ll}
0 & \sigma \\
\frac{1}{\sigma} & 0
\end{array}\right) .
\end{aligned}
$$

Here $\sigma=\sqrt{k^{2}-w^{2}}$ and $I$ is the identity matrix. Furthermore, the matrix

$$
C(w)=\left(\begin{array}{cc}
0 & \sigma \\
\frac{1}{\sigma} & 0
\end{array}\right)
$$

satisfies the relation $C^{2}(w)=I$. Accordingly, instead of decomposing $A(w)$ multiplicatively, we decompose $B(w)$ additively, such that $B_{+}(w)+B_{-}(w)=$ $B(w)$ where $B_{+}(w)$ is analytic in the upper half-plane $\operatorname{Im} w>-\operatorname{Im} k$ and $B_{-}(w)$ is analytic in the lower half-plane $\operatorname{Im} w<\operatorname{Im} k$. Now we have that

$$
B_{+}(w)=I \ln \sqrt{2}-\frac{\mathrm{i} \pi}{4}\left[\begin{array}{cc}
0 & \sigma \\
\frac{1}{\sigma} & 0
\end{array}\right] \frac{2}{\pi} \arctan \sqrt{\frac{k-w}{k+w}}
$$

and $\quad B_{-}(w)=-\frac{\mathrm{i} \pi}{4}\left(\begin{array}{cc}0 & \sigma \\ \frac{1}{\sigma} & 0\end{array}\right) \frac{2}{\pi} \arctan \sqrt{\frac{k+w}{k-w}}$.

This decomposition is straightforward since it has already appeared in some earlier work [3]. We have here

$$
\frac{1}{\sigma}=\frac{2}{\pi \sigma} \arctan \sqrt{\frac{k+w}{k-w}}+\frac{2}{\pi \sigma} \arctan \sqrt{\frac{k-w}{k+w}}
$$

where the first term is analytic in the lower half-plane $\operatorname{Im} w<\operatorname{Im} k$, while the second one is analytic in the upper half-plane $\operatorname{Im} w>-\operatorname{Im} k$. The function $\sigma$ may be rewritten $\sigma^{2} / \sigma$ and since $\sigma^{2}$ is regular, may be dealt with similarly. We note that there is no problem concerning the commutativity of $I$ and $C(w)$. Hence

$$
A_{+}(w)=\exp \left[I \ln \sqrt{2}-\frac{\mathrm{i}}{2} C(w) \arctan \sqrt{\frac{k-w}{k+w}}\right]
$$


and $\quad A_{-}(w)=\exp \left[-\frac{\mathrm{i}}{2} C(w) \arctan \sqrt{\frac{k+w}{k-w}}\right]$.

Now $A_{+}(w)$ can be reduced to

$$
\begin{aligned}
A_{+}(w)= & \sqrt{2} I\left[I \cos \left(\frac{1}{2} \arctan \sqrt{\frac{k-w}{k+w}}\right)\right. \\
& \left.-\mathrm{i} C(w) \sin \left(\frac{1}{2} \arctan \sqrt{\frac{k-w}{k+w}}\right)\right] \\
\text { and } A_{-}(w)= & {\left[I \cos \left(\frac{1}{2} \arctan \sqrt{\frac{k+w}{k-w}}\right)\right.} \\
& \left.-\mathrm{i} C(w) \sin \left(\frac{1}{2} \arctan \sqrt{\frac{k+w}{k-w}}\right)\right] .
\end{aligned}
$$

$A_{+}(w)$ and $A_{-}(w)$ clearly commute and indeed may be simplified by using some elementary trigonometric identities. We have then

$$
A_{+}(w)=\sqrt{2}\left[I \sqrt{\frac{\sqrt{2 k}+\sqrt{k+w}}{2 \sqrt{2 k}}}-\mathrm{i} C(w) \sqrt{\frac{\sqrt{2 k}-\sqrt{k+w}}{2 \sqrt{2 k}}}\right]
$$

and $A_{-}(w)=\left[I \sqrt{\frac{\sqrt{2 k}+\sqrt{k-w}}{2 \sqrt{2 k}}}-\mathrm{i} C(w) \sqrt{\frac{\sqrt{2 k}-\sqrt{k-w}}{2 \sqrt{2 k}}}\right]$.

Written out in full, we get

$$
\begin{aligned}
& A_{+}(w)=\sqrt{2}\left[\begin{array}{cc}
\sqrt{\frac{\sqrt{2 k}+\sqrt{k+w}}{2 \sqrt{2 k}}} & -\mathrm{i} \sigma \sqrt{\frac{\sqrt{2 k}-\sqrt{k+w}}{2 \sqrt{2 k}}} \\
-\frac{\mathrm{i}}{\sigma} \sqrt{\frac{\sqrt{2 k}-\sqrt{k+w}}{2 \sqrt{2 k}}} & \sqrt{\frac{\sqrt{2 k}+\sqrt{k+w}}{2 \sqrt{2 k}}}
\end{array}\right] \\
& A_{-}(w)=\left(\begin{array}{cc}
\sqrt{\frac{\sqrt{2 k}+\sqrt{k-w}}{2 \sqrt{2 k}}}-\mathrm{i} \sigma \sqrt{\frac{\sqrt{2 k}-\sqrt{k-w}}{2 \sqrt{2 k}}} \\
-\frac{\mathrm{i}}{\sigma} \sqrt{\frac{\sqrt{2 k}-\sqrt{k-w}}{2 \sqrt{2 k}}} & \sqrt{\frac{\sqrt{2 k}+\sqrt{k-w}}{2 \sqrt{2 k}}}
\end{array}\right)
\end{aligned}
$$

and $A_{-}(w)=$ 
It is evident that $A_{-}(w)$ and $A_{+}(w)$ commute. It is not obvious that $A_{-}(w) A_{+}(w)=A(w)$ when written in the above form, although it is obvious when written in the trigonometric form as it originally appears. In order to show that $A_{-}(w) A_{+}(w)=A(w)$, we need only demonstrate that

$$
\begin{aligned}
& \sqrt{\frac{\sqrt{2 k}+\sqrt{k+w}}{2 \sqrt{2 k}}} \sqrt{\frac{\sqrt{2 k}+\sqrt{k-w}}{2 \sqrt{2 k}}} \\
- & \sqrt{\frac{\sqrt{2 k}-\sqrt{k+w}}{2 \sqrt{2 k}}} \sqrt{\frac{\sqrt{2 k}-\sqrt{k-w}}{2 \sqrt{2 k}}} \\
+ & \sqrt{\frac{\sqrt{2 k}+\sqrt{k+w}}{2 \sqrt{2 k}}} \sqrt{\frac{\sqrt{2 k}-\sqrt{k-w}}{2 \sqrt{2 k}}} \\
+ & \sqrt{\frac{\sqrt{2 k}-\sqrt{k+w}}{2 \sqrt{2 k}}} \sqrt{\frac{\sqrt{2 k}+\sqrt{k-w}}{2 \sqrt{2 k}}}
\end{aligned}
$$

are constant and equal to $1 / \sqrt{2}$. To accomplish this, we merely need differentiate these expression with respect to $w$ and it turns out that their derivatives vanish. Hence the above functions are constants and they can be determined by evaluating them at $w=0$.

Now we shall verify that $A_{+}(w)$ has analytic elements in the upper halfplane, and that det $A_{+}(w)$ is analytic and doesn't vanish there. This latter requirement is needed since we shall have need for $A_{+}^{-1}(w)$ in our subsequent development. First we note that det $A_{+}(w)=\sqrt{2}$, so that this condition is fulfilled. As for the elements of $A_{+}(w)$, the main diagonal elements are clearly analytic in the upper half-plane $\operatorname{Im} w>-\operatorname{Im} k$. The off-diagonal elements can be rewritten to show this. For example

$$
\sigma \sqrt{\frac{\sqrt{2 k}-\sqrt{k+w}}{2 \sqrt{2 k}}}=\frac{(k-w) \sqrt{k+w}}{\sqrt{2 \sqrt{2 k}(\sqrt{2 k}+\sqrt{k+w})}}
$$

which is also analytic in the upper half-plane $\operatorname{Im} w>-\operatorname{Im} k$. Similar comments may be made about $A_{-}(w)$.

\section{The Matrix Wiener-Hopf Separation}

Having factored the matrix $A(w)$, we can now write equation (2.3) as

$$
\begin{aligned}
& A_{+}(w) A_{-}(w) \hat{f}(w)-\frac{2}{\left(w-k_{x}\right)}\left(\begin{array}{c}
k_{y} \\
-\mathrm{i}
\end{array}\right)=-2 \hat{F}(w) \\
& A_{-}(w) \hat{f}(w)-\frac{2 A_{+}^{-1}(w)}{\left(w-k_{x}\right)}\left(\begin{array}{c}
k_{y} \\
-\mathrm{i}
\end{array}\right)=-2 A_{+}^{-1}(w) \hat{F}(w) .
\end{aligned}
$$


The middle term can be decomposed additively into two terms, one of which is analytic in the lower half-plane $\operatorname{Im} w<\operatorname{Im} k_{x}$ while the other is analytic in the upper half-plane $\operatorname{Im} w>-\operatorname{Im} k$. This gives us

$$
\begin{aligned}
& A_{-}(w) f(w)-\frac{2 A_{+}^{-1}\left(k_{x}\right)}{\left(w-k_{x}\right)}\left(\begin{array}{c}
k_{y} \\
-\mathrm{i}
\end{array}\right) \\
& =\frac{2\left[A_{+}^{-1}(w)-A_{+}^{-1}\left(k_{x}\right)\right]}{\left(w-k_{x}\right)}\left(\begin{array}{c}
k_{y} \\
-\mathrm{i}
\end{array}\right)-2 A_{+}^{-1}(w) \hat{F}(w) .
\end{aligned}
$$

The left side of equation (3.2) is analytic in the lower half-plane $\operatorname{Im} w<\operatorname{Im} k_{x}$ (as well as the determinant of $A_{-}(w)$ ), while the right side is analytic in the upper half-plane $\operatorname{Im} w>-\operatorname{Im} k$ (as well as the determinant of $A_{+}(w)$ ) and both sides are analytic in the common strip $-\operatorname{Im} k<\operatorname{Im} w<\operatorname{Im} k_{x}$. The left side is therefore the analytic continuation of the right side and is therefore analytic everywhere. Hence

$$
A_{-}(w) \hat{f}(w)-\frac{2 A_{+}^{-1}\left(k_{x}\right)}{\left(w-k_{x}\right)}\left(\begin{array}{c}
k_{y} \\
-\mathrm{i}
\end{array}\right)=\left(\begin{array}{l}
\tilde{\sigma}_{1}(w) \\
\delta_{2}(w)
\end{array}\right)
$$

and

$$
\frac{2\left[A_{+}^{-1}(w)-A_{+}^{-1}\left(k_{x}\right)\right]}{\left(w-k_{x}\right)}\left(\begin{array}{c}
k_{y} \\
-\mathrm{i}
\end{array}\right)-2 A_{+}^{-1}(w) \hat{F}(w)=\left(\begin{array}{l}
\delta_{1}(w) \\
f_{2}(w)
\end{array}\right)
$$

where $\mathscr{E}_{1}(w)$ and $\mathscr{E}_{2}(w)$ are entire functions.

In order to determine the entire functions $\delta_{1}(w)$ and $\delta_{2}(w)$, we need the edge condition for $f_{1}(x), f_{2}(x), F_{1}(x), F_{2}(x)$ and the asymptotic behavior of $A_{-}(w)$ and $A_{+}(w)$ for $|w| \rightarrow \infty$, Im $w$ suitably limited. The edge condition will provide the asymptotic behavior of $\hat{f}_{1}(w), \hat{f}_{2}(w), \hat{F}_{1}(w)$ and $\hat{F}_{2}(w),|w| \rightarrow \infty$, $\operatorname{Im} w$ suitably limited. We note that $f_{1}(x)=O\left(x^{-3 / 4}\right), x \rightarrow+0$ and $f_{2}(x)=$ $O\left(x^{1 / 4}\right), x \rightarrow+0$. With this information and the fact that $f_{1}(w)$ and $f_{2}(w)$ exist in the lower half-plane $\operatorname{Im} w<\operatorname{Im} k_{x}$, we have that $f_{1}(w)=O\left(w^{-1 / 4}\right)$ and $f_{2}(w)$ $=O\left(w^{-5 / 4}\right),|w| \rightarrow \infty, \operatorname{Im} w<\operatorname{Im} k_{x}$. Therefore $\delta_{1}(w)$ is bounded while $\tilde{E}_{2}(w) \rightarrow 0$. Since $F_{1}(x)$ and $F_{2}(x), x \rightarrow-0$ have similar edge behavior, we can assert that $\delta_{1}(w)$ is bounded in the upper half-plane $\operatorname{Im} w>-\operatorname{Im} k,|w| \rightarrow \infty$ and $\mathscr{E}_{2}(w) \rightarrow 0$ there. Hence by the Liouville theorem, $\delta_{1}(w)=c(c$ a constant $)$ and $\tilde{\delta}_{2}(w) \equiv 0$. We can therefore solve for $f(w)$ to obtain

$$
f(w)=\frac{2 A_{-}^{-1}(w) A_{+}^{-1}\left(k_{x}\right)}{\left(w-k_{x}\right)}\left(\begin{array}{c}
k_{y} \\
-\mathrm{i}
\end{array}\right)+A_{-}^{-1}(w)\left(\begin{array}{l}
c \\
0
\end{array}\right) .
$$

\section{The Determination of the Arbitrary Constant $c$}

The constant $c$ can be determined by noting that the vector $\hat{f}(w)$ does not satisfy the Riemann-Lebesgue lemma unless the constant $c$ is chosen appropriately. Hence we shall examine (3.5) in component form. We have 


$$
\begin{aligned}
f(w)= & \frac{\sqrt{2}}{\left(w-k_{x}\right)}\left[\begin{array}{cc}
\cos D_{-}(w) & \mathrm{i} \sigma \sin D_{-}(w) \\
\frac{\mathrm{i} \sin D_{-}(w)}{\sigma} & \cos D_{-}(w)
\end{array}\right] \\
& \times\left(\begin{array}{c}
k_{y}\left[\cos D_{+}\left(k_{x}\right)+\sin D_{+}\left(k_{x}\right)\right] \\
i\left[\sin D_{+}\left(k_{x}\right)-\cos D_{+}\left(k_{x}\right)\right]
\end{array}\right)+c\left[\begin{array}{c}
\cos D_{-}(w) \\
\frac{\mathrm{i} \sin D_{-}(w)}{\sigma}
\end{array}\right]
\end{aligned}
$$

where $D_{-}(w)=\frac{1}{2} \arctan \sqrt{\frac{k+w}{k-w}}$ and $D_{+}(w)=\frac{1}{2} \arctan \sqrt{\frac{k-w}{k+w}}$.

In component form we have

$$
\begin{aligned}
f_{1}(w)= & \frac{\sqrt{2}}{\left(w-k_{x}\right)}\left\{\begin{array}{l}
k_{y} \cos D_{+}(w)\left[\cos D_{+}\left(k_{x}\right)+\sin D_{+}\left(k_{x}\right)\right] \\
-\sigma \sin D_{-}(w)\left[\sin D_{+}\left(k_{x}\right)-\cos D_{+}\left(k_{x}\right)\right]
\end{array}\right\} \\
& +c \cos D_{-}(w)
\end{aligned}
$$

and

$$
\begin{aligned}
f_{2}(w)= & \frac{\sqrt{2}}{\left(w-k_{x}\right)}\left\{\begin{array}{l}
\frac{\mathrm{i} k_{y}}{\sigma} \sin D_{-}(w)\left[\cos D_{+}\left(k_{x}\right)+\sin D_{+}\left(k_{x}\right)\right] \\
-\mathrm{i} \cos D_{-}(w)\left[\sin D_{+}\left(k_{x}\right)-\cos D_{+}\left(k_{x}\right)\right]
\end{array}\right\} \\
& +\frac{\mathrm{ic} \cos D_{-}(w)}{\sigma}
\end{aligned}
$$

We examine (4.1) and note that $\hat{f}_{1}(w)=O\left(w^{1 / 4}\right),|w| \rightarrow \infty, \operatorname{Im} w<\operatorname{Im} k_{x}$. Since $f_{1}(w)$ is a classical unilateral Fourier transform, this cannot come to pass and we therefore must choose the constant $c$ to eliminate this behavior. Upon noting the form of the sine and cosine of $D_{-}(w)$, we see this forbidden behavior comes from the following two terms:

$$
\begin{aligned}
& \frac{-\sqrt{2} \sigma \sin D_{-}(w)}{\left(w-k_{x}\right)}\left[\sin D_{+}\left(k_{x}\right)-\cos D_{+}\left(k_{x}\right)\right]+c \cos D_{-}(w) \\
& =\frac{-\sqrt{2} \sqrt{k^{2}-w^{2}}}{\left(w-k_{x}\right)} \sqrt{\frac{\sqrt{2 k}-\sqrt{k-w}}{2 \sqrt{2 k}}}\left[\sin D_{+}\left(k_{x}\right)-\cos D_{+}\left(k_{x}\right)\right] \\
& +c \sqrt{\frac{\sqrt{2 k}-\sqrt{k+w}}{2 \sqrt{2 k}}} .
\end{aligned}
$$


Hence if we choose $c=\sqrt{2}\left[\sin D_{+}\left(k_{x}\right)-\cos D_{+}\left(k_{x}\right)\right]$ we eliminate the term $O\left(w^{1 / 4}\right),|w| \rightarrow \infty, \operatorname{Im} w<\operatorname{Im} k_{x}$. Upon simplifying (4.1) and (4.2) with this constant, we obtain

(4.3) $f_{1}(w)=$

$$
f_{1}(w)=\frac{(k / 2)^{1 / 4}\left(\sin \frac{\alpha}{4}-\cos \frac{\alpha}{4}\right)(\sqrt{k-w}+\sqrt{2 k})^{1 / 2}}{\sqrt{k-w}-\sqrt{2 k} \sin \frac{\alpha}{2}}
$$

and

(4.4) $\hat{f}_{2}(w)=$

$$
\frac{i(2 k)^{1 / 4}\left(\sin \frac{\alpha}{4}-\cos \frac{\alpha}{4}\right)}{\sqrt{k-w}(\sqrt{2 k}+\sqrt{k-w})^{1 / 2}\left(\sqrt{k-w}+\sqrt{2 k} \sin \frac{\alpha}{2}\right)} .
$$

Clearly $\hat{f}_{1}(w)=O\left(w^{-1 / 4}\right)$, and $\hat{f}_{2}(w)=O\left(w^{-5 / 4}\right),|w| \rightarrow \infty, \operatorname{Im} w<\operatorname{Im} k_{x}$ and therefore $f_{1}(x)=O\left(x^{-3 / 4}\right)$ and $f_{2}(x)=O\left(x^{1 / 4}\right), x \rightarrow+0$. The angle $\alpha$ defines the direction of propagation of the incident plane wave and $0<\alpha<\pi$.

\section{References}

[1] Daniele, V. G.: On the Factorization of Wiener-Hopf Matrices in Problems Solvable with Hurd's Method I.E.E.E. Transactions on Astennas and Propagation, AP-26 (1978) 614-616

[2] Heins, A. E.: Systems of Wiener-Hopf Integral Equations and their Application to some Boundary Value Problems in Electromagnetic Theory. Proc. of Symposia in Appl. Math. Vol. II (1950) $76-81$

[3] Heins, A. E.: The Radiation and Transmission Properties of a Pair of Parallel Plates. Quart. Appl. Math. 6 (1948) 215-220

[4] Heins, A. E.: The Sommerfeld Half-Plane Problem Revisited I. Math. Meth. in the Appl. Sci. 4 (1982) $74-90$

[5] Hurd, R. A.: The Wiener-Hopf-Hilbert Method for Diffraction Problems. Can J. Phys. 54 (1976) $775-780$

[6] Hurd, R. A.; Przeździecki, S.: Diffraction by a Half-plane Perpendicular to the Distinguished Axis of a Gyrotropic Medium. J. Math. Phys. 17 (1976) $1838-1847$

Albert E. Heins

Department of Mathematics

The University of Michigan

Ann Arbor, MI 48109, USA

(Received August 26, 1981) 